\title{
Réceptivité expérimentale à l'infestation par les larves de Protostrongylidés de quelques Hélicides fréquents au Maroc
}

\author{
Facteurs de variation
}

\author{
par J. CABARET \\ Institut agronomique et vétérinaire Hassan-II, Département de Parasitologie. \\ B.P. 704 Rabat - Agdal
}

\section{Résumé.}

Divers Hélicidés habituellement présents sur les pâturages dans la région de Rabat ont été infestés par un mélange plurispécifique de larves de Protostrongylidés. L'origine pulmonaire ou fécale des larves ne semble pas influencer leur pouvoir infestant pour les Mollusques. L'âge des larves utilisées pour l'infestation joue un rôle important sur le futur degré d'infestation des Mollusques: les larves jeunes sont plus infestantes. Les Hélicidés les plus réceptifs à l'infestation sont par ordre décroissant: Otala lactea, Cochlicella conoidea, C. acuta et Euparypha pisana, Helicella gigaxii. O. lactea et E. pisana ont permis le développement de $M$. capillaris, $C$. nigrescens et $N$. linearis. La première espèce est un nouvel hôte intermédiaire. Les deux espèces de Cochlicella ne sont pas un hôte intermédiaire pour $M$. capillaris. Les Hélicidés assurent la régulation tant quantitative que spécifique de l'infestation des Ovins.

\section{Summary.}

Receptivity of some Helicids frequent in Morocco to the infestation by the larvae of Protostongylids. Factors of variation.

Several species of Helicids common on pasture in the Rabat area were infected by a plurispecific mixture of larvae of Protostrongylids. The fecal or pulmonary origin of the

Accepté le 15 mai 1979. 
larvae does not seem to influence their infective ability. The age of the larvae used for infection displays an important role on the future degree of infection of Molluscs : the young larvae are more infestive. The Helicids that were most receptive to infection are, arranged in a decreasing order: Otala lactea, Cochlicella conoidea, C. acuta and Euparypha pisana, Helicella gigaxii. O. lactea and E. pisana permitted the development of Muellerius capillaris, Cystocaulus nigrescens and Neostrongylus linearis. The former species of Helicid is a new intermediary host. The two species of Cochlicella were not suitable for M. capillaris. The Helicids ensure the quantitative and specific regulation of the infection of the Ovines.

Les protostrongylidoses constituent une dominante pathologique chez les Ovins au Maroc, comme l'attestent les travaux de Joyeux et Gaud, 1946 ; Alahkam, 1977 ; Bahaïda, 1978. La faune protostrongylidienne est représentée par Muellerius capillaris, Neostrongylus linearis, Cystocaulus nigrescens et Protostrongylus sp.; l'espèce prédominante est $M$. capillaris (Bahaïda, 1978).

Les Hélicidés que nous avons recensés tout au long de l'année sur les pâturages de la région de Rabat sont : Cochlicella acuta, C. ventricosa, C. conoidea, Euparypha pisana, Otala lactea, Helicella sp.

L'espèce de mollusque considérée joue un rôle fondamental dans la réceptivité à l'infestation par les Protostrongylidés selon la revue de Kassai, 1957. Ce rôle s'exerce sur la réceptivité totale (nombre de larves par Mollusque) et sur la réceptivité particulière à une espèce de Protostrongylidé. Ainsi Cabaret et Dakkak, 1979, ont montré que chez Cochlicella ventricosa, $M$. capillaris ne se développe pas alors que $N$. linearis est retrouvé au stade $\mathrm{L}_{3}$ au bout de 2 semaines et $C$. nigrescens au bout de 3 semaines.

L'origine fécale ou pulmonaire est un facteur à considérer: les larves d'origine pulmonaire auraient un pouvoir infestant plus faible selon Babos, 1961 ; Nürnberg, 1961 ; Ramirez-Fernandez, 1967. Enfin, l'âge des larves $\mathrm{L}_{1}$ devrait vraisemblablement exercer une influence sur la réceptivité.

Le but de notre travail est d’apprécier expérimentalement la réceptivité des Hélicidés les plus fréquemment rencontrés sur les pâturages de la région de Rabat. Cette mesure a été réalisée sur des spécimens prélevés en automne. L’appréciation de cette réceptivité a été réalisée en relation avec deux facteurs de variation liés aux larves elles-mêmes : leur âge et leur origine fécale ou pulmonaire.

\section{Matériel et méthode}

\section{Larves $\mathrm{L}_{1}$ de Protostrongylidés.}

La composition du mélange plurispécifique issu de fèces est : $M$. capillaris $(64,7$ p. cent), $N$. linearis $(23,5$ p. cent) et $C$. nigrescens $(11,8$ p. cent). La composition du mélange issu de poumons est pour les mêmes espèces respectivement de 74,4,7,0, 18,6 p. cent. 


\section{Hélicidés.}

Ils proviennent d'une parcelle indemne de Protostrongylidés. Les exemplaires de Cochlicella acuta, C. conoidea, Euparypha pisana, Helicella gigaxii sont adultes ; ceux de Otala lactea sont constitués de jeunes et d'adultes. Ceci correspond approximativement à l'état de la faune malacologique que l'on peut récolter en novembre dans la région de Rabat.

\section{Infestation et entretien des Hélicidés.}

Ils sont réalisés suivant les modalités exposées par Cabaret et Dakkak, 1979. La durée du contact entre les Mollusques et les larves $\mathrm{L}_{1}$ est de une demi-heure.

\section{Technique de digestion des Mollusques.}

Les larves des différents stades sont comptées trois semaines après l'infestation, dans le liquide de digestion pepsique. Les Mollusques entiers sont dilacérés en petits fragments par écrasement pour les petites espèces ou avec des ciseaux pour les plus grosses. Le broyat est additionné de 5 parties du liquide de digestion suivant : pepsine (30000 E/g) 1,3 g ; $\mathrm{HCl}$ fumant $2 \mathrm{ml}$; eau ordinaire $100 \mathrm{ml}$. La proportion d'acide chlorhydrique est plus importante que celle utilisée habituellement pour deux raisons : les Mollusques sécrètent à l'agonie une quantité abondante de mucus basique $(\mathrm{pH}$ : 7 à 8,5 selon les espèces) et certaines espèces hébergent de nombreux Nématodes saprophytes selon Chitwood et Chitwood, 1937, qui gênent la lecture et sont partiellement détruits par une acidité importante. L'incubation dure au moins 3 heures à $40{ }^{\circ} \mathrm{C}$.

\section{Méthodes d'analyse des données.}

Elles sont le plus souvent non paramétriques, les données ne présentant pas les caractères d'hétéroscédacité et de normalité. Les tests utilisés sont décrits par Siegel, 1956.

Le coefficient $k$ de Neymann a été utilisé pour mesurer la dispersion; sa définition et les méthodes de comparaison de ce coefficient sont exposées dans Southwood, 1971.

\section{Résultats et analyse}

\section{Réceptivité et origine des larves.}

Les résultats présentés dans le tableau I montrent qu'il n'existe pas de différence sensible dans les degrés d'infestations de $C$. conoidea selon l'origine pulmonaire ou fécale des larves $\mathrm{L}_{1}$. L'écart observé dans les degrés d'infestation d'O. lactea est dû à l'existence d'une valeur extrême (34) chez un individu. 
Table I. - Degré d'infestation de deux espèces de mollusques en fonction de l'origine des larves $L_{1}$.

\begin{tabular}{|c|c|c|c|c|c|}
\hline $\begin{array}{c}\text { Espèce } \\
\text { de } \\
\text { mollusques }\end{array}$ & $\begin{array}{c}\text { Nombre } \\
\text { de } \\
\text { mollusques }\end{array}$ & $\begin{array}{c}\text { Dose } \\
\text { moyenne } \\
\text { infestante } \\
\text { par individu }\end{array}$ & $\begin{array}{c}\text { Age } \\
\text { des larves } \\
\text { en jours }\end{array}$ & $\begin{array}{c}\text { Degré } \\
\text { d'infestation } \\
\text { moyen }\end{array}$ & $\begin{array}{c}\text { Valeurs } \\
\text { extrêmes } \\
\text { du degré } \\
\text { d'infestation }\end{array}$ \\
\hline \multirow[t]{2}{*}{ C. conoidea ............... } & 4 & $\begin{array}{c}40 \\
\text { (poumons) }\end{array}$ & 3 & 0,5 & $0 \cdot 1$ \\
\hline & 9 & $\begin{array}{c}40 \\
\text { (féces) }\end{array}$ & 5 & 0,7 & $0-2$ \\
\hline \multirow[t]{2}{*}{ O. lactea adultes ......... } & 3 & $\begin{array}{c}300 \\
\text { (poumons) }\end{array}$ & 7 & 11,6 & 034 \\
\hline & 3 & $\begin{array}{c}150 \\
\text { (fẻces) }\end{array}$ & 6 & 4,0 & $0-10$ \\
\hline
\end{tabular}

\section{Réceptivité et âge des larves $\mathrm{L}_{1}$.}

Le tableau II concerne l'infestation de $C$. conoidea (40 larves/Mollusque) et le tableau III celle d'O. lactea (300 larves/Mollusque). Dans le premier cas, les larves extraites de fèces ont été stockées, entre les diverses infestations, à $4^{\circ} \mathrm{C}$; celles qui ont servi à l'infestation de $O$. lactea ont été conservées à $18^{\circ} \mathrm{C}$. Pour ce dernier, un seul individu adulte a été utilisé pour chaque mesure.

Tableau II. - Degré d'infestation de C. conoidea en fonction de l'âge des larves L I utilisées.

\begin{tabular}{|c|c|c|c|c|}
\hline Ages des larves en jours & 5 & 12 & 19 & 26 \\
\hline Degré d'infestation moyen ...................... & 0,55 & 0,49 & 0,16 & 0,05 \\
\hline Valeurs extrêmes ................................. & $0-2$ & $0-3$ & $0-2$ & $0-1$ \\
\hline 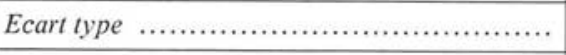 & 0,80 & 0,80 & 1,6 & 1,5 \\
\hline Coefficient $k$ de Neymann $\quad . . . \ldots \ldots \ldots \ldots \ldots \ldots$ & 3,36 & 1,60 & 0,01 & 0,001 \\
\hline Taille du lot .................... & 9 & 18 & 18 & 18 \\
\hline
\end{tabular}

Tableau III. - Degré d'infestation de Otala lactea en fonction de l'âge des larves L1 utilisées.

\begin{tabular}{|c|c|c|c|c|c|c|c|c|}
\hline $\begin{array}{c}\text { Age des larves } \\
\text { en jours }\end{array}$ & 2 & 4 & 6 & 8 & 11 & 14 & 18 & 25 \\
\hline Degré d'infestation & 18 & 10 & 0 & 2 & 0 & 1 & 0 & 0 \\
\hline
\end{tabular}

Le pouvoir infestant des larves $\mathrm{L}_{1}$ diminue avec l'âge. Cette diminution est lente à s'établir pour une température de conservation basse $\left(4^{\circ} \mathrm{C}\right)$; elle s'accélère rapidement dès la troisième semaine (tableau II) chez C. conoidea. L'évolution du pou- 
voir infestant mesuré chez $O$. lactea présente la même tendance pour des larves conservées à la température ambiante ; le processus est cependant accéléré et un pouvoir infestant élevé n'est conservé que pendant les quatre premiers jours. La diminution du pouvoir infestant des larves $\mathrm{L}_{1}$ récoltées dans les fèces s'ajuste à une loi exponentielle négative en fonction de leur âge $t$, en jours :
C. conoidea
$\mathrm{L}_{3}=1,145 e-0^{\prime} 110 t$
$\mathrm{P}<0,05$
O. lactea
$\left(\mathrm{L}_{3}+1\right)=7,90 e^{-0^{\prime} 106 t} \quad \mathrm{P}=0,05$

avec: $\mathrm{L}_{3}$ : nombre de larves du troisième stade récoltées 3 semaines après l'infestation.

\section{Réceptivité comparée des différentes espèces de Mollusques.}

Les degrés d'infestation des espèces d'Hélicidés considérées (tableau IV), après une infestation à la dose de 300 larves/mollusques, diffèrent significativement dans

Tableau IV. - Réceptivité comparée de quelques Hélicidés, trois semaines après l'infestation.

\begin{tabular}{|c|c|c|c|c|c|c|}
\hline \multirow{2}{*}{$\begin{array}{c}\text { Espèce } \\
\text { de } \\
\text { mollusque }\end{array}$} & \multirow{2}{*}{$\begin{array}{l}\text { Nombre } \\
\text { d'individus } \\
\text { infestes }\end{array}$} & \multirow{2}{*}{$\begin{array}{c}\text { Degré } \\
\text { d'infestation } \\
\text { moyen } \\
\text { (L-3/mollusq.) }\end{array}$} & \multirow{2}{*}{$\begin{array}{l}\text { Larves } \\
\text { mortes } \\
\text { p. cent }\end{array}$} & \multicolumn{3}{|c|}{$\begin{array}{l}\text { Proportion des différentes espèces } \\
\text { de Protostrongylidés }\end{array}$} \\
\hline & & & & $\begin{array}{l}M . \\
\text { capillaris }\end{array}$ & $\begin{array}{l}\text { C. } \\
\text { nigrescens }\end{array}$ & $\begin{array}{l}N . \\
\text { linearis }\end{array}$ \\
\hline C. acuta .................. & 8 & 2,87 & 4 & 0 & 56 & 44 \\
\hline C. conoidea ................ & 8 & 4,25 & 6 & 0 & 34 & 66 \\
\hline E. pisana ................. & 8 & 2,12 & 20 & 33 & 27 & 40 \\
\hline H. gigaxii $\ldots \ldots \ldots \ldots \ldots \ldots$ & 5 & 0,40 & 50 & 0 & 0 & 100 \\
\hline O. lactea $\ldots \ldots \ldots \ldots \ldots$ & 11 & 6,54 & 15 & 39 & 34 & 27 \\
\hline
\end{tabular}

leur ensemble (test de Kruskall et Wallis : P $<0,05$ ). L'utilisation du test de Wald et Wolfowitz pour les comparaisons deux à deux permet de présenter l'échelle de réceptivité croissante: Helicella gigaxii, Cochlicella acuta et Euparypha pisana, Cochlicella conoidea, Otala lactea. Le nombre de larves mortes est particulièrement bas chez les deux espèces de Cochlicella; elles étaient pratiquement toutes au stade jeune $\mathrm{L}_{2}$.

O. lactea et E. pisana conviennent au développement des trois espèces de Protostrongylidés présentes. Les deux espèces de Cochlicella ne permettent le développement que de $N$. linearis et de $C$. nigrescens. Aucune conclusion ne peut être fournie pour $H$. gigaxii étant donné la faiblesse du nombre de larves récoltées. Dans aucune des espèces de Mollusques infestés ne se retrouvent les proportions initiales du mélange infestant: $M$. capillaris est toujours sous-représenté à l'inverse de $N$. linearis et C. nigrescens. 


\section{Discussion}

\section{Facteurs de variation de la réceptivité des Mollusques.}

L'origine des larves $\mathrm{L}_{1}$ utilisées pour l'infestation, contrairement aux opinions de Nürnberg, 1961, et Ramirez-Fernandez, 1967, ne semble pas avoir une importance marquée sur leur évolution ultérieure chez le Mollusque hôte intermédiaire. Le transit des larves $\mathrm{L}_{1}$ dans le tube digestif des ovins n'est donc pas une étape aboslument nécessaire à l'acquisition du pouvoir infestant.

La réceptivité moyenne à l'infestation par les Protostrongylidés d'une espèce de Mollusque donnée est fonction de l'âge des larves $\mathrm{L}_{1}$. Ce facteur permet d'expliquer $94 \%\left(r^{2}=0,94\right)$ de la réceptivité moyenne de Cochlicella conoidea. La relation existant entre l'âge des larves et la réceptivité moyenne est du type : $\mathrm{L}_{3}=\mathrm{L}_{\max } e^{-r}$, avec $\mathrm{L}_{3}$ : nombre de larves $\mathrm{L}_{1}$ qui ont évolué en $\mathrm{L}_{3} ; \mathrm{L}_{\max }$ : nombre maximal de $\mathrm{L}_{3}$ que l'on peut obtenir chez une espèce particulière de Mollusque pour une certaine dose infestante ; $r$ : coefficient de décroissance du pouvoir infestant. Ce coefficient $r$ semble peu affecté par l'espèce de Mollusque réceptrice et par la température (cf. formules 1 et 2 ).

La réceptivité individuelle des Mollusques à l'infestation n'est pas régulière, ce qui se traduit par une loi de distribution variable de la population des $\mathrm{L}_{3}$. La nature de cette loi peut être appréciée au moyen du coefficient d'aggrégation $k$ de Neymann qui passe de 3,36 pour des larves âgées de 5 jours à 0,001 pour des larves âgées de 26 jours (tableau II). Les divers coefficients sont significativement différents. L'agrégation de la distribution est d'autant plus forte que la valeur du coefficient $k$ est faible (inférieur à 1); il s'agit alors d'une loi binomiale négative et les larves ont tendance à s'accumuler chez certains Mollusques. Lorsque le coefficient $k$ a une valeur élevée, la population suit une loi de Poisson, ce qui est le cas pour les larves âgées de 5 à 12 jours. La distribution des larves $\mathrm{L}_{3}$ suit une loi de Poisson lorsque les larves $\mathrm{L}_{1}$ ayant servi à l'infestation sont jeunes et une loi binomiale négative lorsque celles-ci sont âgées. Les variations de réceptivité individuelle des Mollusques seront donc importantes avec des larves $\mathrm{L}_{1}$ âgées.

Les résultats concernant la réceptivité des Hélicidés étudiés aux trois espèces de Protostrongylidés sont en accord avec les travaux de Kassai, 1957, à l'exception de ceux sur Cochlicella. En effet, selon cet auteur, C. acuta est réceptrice à l'infestation par $M$. capillaris. La discordance entre ces résultats est peut-être due au fait que l'évolution n'a pas été suivie jusqu'au stade $\mathrm{L}_{3}$; nous avons ainsi observé une larve $\mathrm{L}_{1}$ morte de $M$. capillaris chez $C$. conoidea. Les résultats obtenus pour l'infestation de Cochlicella en ce qui concerne $C$. nigrescens et $N$. linearis correspondent à ceux obtenus par Ramirez-Fernandez, 1967, Cabaret et Dakkak, 1979. Otala lactea est un nouvel hôte pour $M$. capillaris, $N$. linearis et $C$. nigrescens. 


\section{Comparaison des réceptivités expérimentales et naturelles.}

Bahaïda, 1978, avec des Mollusques prélevés en automne 1977 sur un pâturage dans la région de Rabat, a présenté l'échelle de réceptivité : Cochlicella sp. et Helicella sp., E. pisana, Otala lactea. L'année suivante, nous avons effectué des prélèvements de $C$. conoidea et de $E$. pisana à la même époque et les résultats ont été similaires. Le classement des Hélicidés paraît donc différent dans les conditions naturelles; Cochlicella sp. en particulier semble être un hôte intermédiaire de moindre importance. Nous avons constaté que les jeunes $C$. conoidea ont tendance à former des grappes en position haute (arbres et arbustes), ce qui rend peu probable leur infestation. Ces grappes, contrairement à celles des autres espèces (Bigot, 1967), se dissocient peu, même lorsqu'il pleut. L'éthologie de $C$. conoidea dans son biotope est sans doute la cause de la faible réceptivité observée en début d'automne.

Les degrés d'infestations au sein des individus d'une espèce sont très variables. Leur loi de distribution s'ajuste à une loi binomiale négative, d'après les données de Bahaïda, 1978. La variabilité individuelle enregistrée pourrait se rapporter soit à la distribution dans l'espace des larves et des Mollusques, soit à des différences de réceptivité individuelle. Elle pourrait aussi être mise en relation avec l'âge des larves $\mathrm{L}_{1}$ qui infestent les Hélicidés, comme le montre notre étude expérimentale.

\section{Les Hélicidés sont des filtres biologiques de l'infestation par les Protostron- gylidés.}

Les Hélicidés assurent une régulation du flux global de larves $\mathrm{L}_{1}$. Ainsi, une infestation de 300 larves $\mathrm{L}_{1}$ /Mollusque permet l'obtention de 0,4 à 6,5 larves/Mollusque (tableau IV). Cette régulation s'exerce au moyen de deux mécanismes différents. Le premier est le phénomène de limitation mis en évidence par Cabaret et Dakkak, 1979, lequel consiste en une faible pénétration des larves $L_{1}$. Le second s'exprime par l'augmentation de la mortalité des stades $\mathrm{L}_{2}$ (tableau IV).

Les Hélicidés régulent également la qualité spécifique du flux de larves $\mathrm{L}_{1}$. Le rendement de l'infestation par $M$. capillaris est particulièrement faible. Le même phénomène est observé dans les conditions naturelles : dans les fèces d'Ovins, l'espèce dominante est $M$. capillaris et à l'intérieur des Mollusques ce sont $N$. linearis et C. nigrescens selon Bahaïda, 1978. Cette régulation pourrait éventuellement compenser les variations de fertilité des diverses espèces de Protostrongylidés et permettre la pérennité de l'infestation des Ovins par les espèces les moins fertiles.

\section{Bibliographie}

Alahkam L. (1977) : Contribution à l'étude parasitologique, épidémiologique et lésionnelle des bronchopneumonies vermineuses du mouton dans la région de Tadla. Thèse Doc. Vét. Rabat.

Babos H. (1961) : Zur Kenntnis der Protostrongylinosen der Leporiden unter besonderer Brücksichtigung der in Ungarn vorkommenden Protostrongylus. Arten. Helmint., 3, 13-37.

Bahaïda B. (1978) : Contribution à l'étude des Protonstrongylidoses du mouton. Production de larves $\mathrm{L}_{1}$ et leur devenir chez les mollusques terrestres hôtes intermédiaires. Thèse Doc. Vét. Rabat. 
Bigot L. (1967): Recherches sur les groupements de gastéropodes terrestres: la constitution des grappes. Vie et Milieu, Série C, 18, 1-27.

Cabaret J., Dakkak A.: Infestation expérimentale de Cochlicella ventricosa par des larves $\mathrm{L}_{1}$ de Protostrongylidés. Ann. Parasitol. Hum. Comp. (sous presse).

Chitwood B. G., Chitwood M.B. (1937): Snails as hosts and carriers of Nematodes and Nematomorpha. The Nautilus, 50, 130-135.

Joyeux C.-H., Gaud J. (1946) : Recherches helminthologiques marocaines. Etude sur la pneumonie vermineuse. Arch. Inst. Past. Maroc, 3, 383-461.

Kassai T. (1957) : Schnecken als zwischenwirte der Protostrongyliden. Z. f. Parasitenkunde, 18, 5-19.

Nürnberg H. (1961) : Dictyocaulus und Protostrongyliden befall bei Schafen. Inaugural Dissertation, Berlin.

Ramirez-Fernandez A.P. (1967) : Epizoologia de las bronconeumonias verminosas ovinas en Léon. Ann. Fac. Vét. Léon, 13, 135-210.

Siegel S. (1956): Non parametric statistics for the behaviourial Sciences. Mc Graw-Hill, Publ., Kogakusha, Tokyo, 312 p.

Southwood T.R. E. (1971): Ecological methods. Chapman and Hall, Publ., London, 391 p. 